Cultures \& Conflits

62 | printemps 2006

Arrêter et juger en Europe

\title{
Généalogie du champ de la coopération judiciaire européenne
}

\section{Antoine Mégie}

\section{OpenEdition}

1 Journals

Édition électronique

URL : http://journals.openedition.org/conflits/2053

DOI : 10.4000/conflits.2053

ISSN : $1777-5345$

Éditeur :

CCLS - Centre d'études sur les conflits lilberté et sécurité, L'Harmattan

Édition imprimée

Date de publication : 1 juin 2006

Pagination : 11-41

ISBN : 2-296-01073-3

ISSN : 1157-996X

Référence électronique

Antoine Mégie, "Généalogie du champ de la coopération judiciaire européenne », Cultures \& Conflits [En ligne], 62 I printemps 2006, mis en ligne le 23 mars 2007, consulté le 30 mars 2021. URL : http:// journals.openedition.org/conflits/2053; DOI : https://doi.org/10.4000/conflits.2053

Ce document a été généré automatiquement le 30 mars 2021.

Creative Commons License 


\title{
Généalogie du champ de la coopération judiciaire européenne
}

\author{
Antoine Mégie
}

« En dépit des apparences, il n'est plus possible aujourd'hui de méconnaître la superposition des normes nationales, régionales et mondiales, ni la surabondance d'institutions et de juges, nationaux et internationaux, à compétence élargie. Ces réalités nouvelles font évoluer le droit vers des systèmes interactifs, complexes et fortement instables. Plus que d'une défaite du droit, c'est d'une mutation qu'il s'agit, dans la conception même de l'ordre juridique ${ }^{1}$ ».

1 Ce phénomène de transformation de l'ordre juridique au niveau international constitue aujourd'hui l'une des dynamiques saillantes qui traversent le droit en tant qu'objet de recherche. Cette évolution a eu pour effet de placer le droit au cœur des modes de régulation entre les différentes échelles de la gouvernance internationale (Etats, communautés régionales, organisations internationales), mais aussi de faire de ce dernier un objet de négociation entre ces niveaux de pouvoir. Le droit pénal - par définition l'un des éléments fondamentaux de la souveraineté des Etats - est aujourd'hui un enjeu de discussions entre les autorités étatiques. A l'échelle européenne, ces discussions ont conduit à l'élaboration du projet de construction " d'un espace de justice, de liberté et de sécurité ».

Depuis une vingtaine d'années dans le cadre européen, la définition du «droit pénal » a évolué d'une activité exclusivement territorialisée à une approche interétatique. Cependant, les transformations qui touchent le domaine pénal concernent actuellement surtout le secteur des procédures pénales. Dans son acceptation la plus large, l'expression « droit pénal » désigne la branche du droit positif qui relève « de la répression par l'Etat des comportements de nature à créer un trouble pour l'ordre social ${ }^{2} »$. De façon théorique, il est possible de décomposer le domaine pénal en trois ensembles : le droit pénal général, le droit pénal spécial et la procédure pénale. Le droit pénal général concerne la structure de l'infraction et les conditions générales de la responsabilité pénale. Il fixe, par ailleurs, l'éventail des diverses peines et mesures de sûreté encourues et énonce les principes selon lesquels ces dernières sont prononcées et appliquées. Le droit pénal spécial représente « le catalogue » des infractions, avec, 
pour chacune, les divers éléments constitutifs et les sanctions applicables. Enfin, la procédure pénale détermine les règles techniques de mise en œuvre de la répression et ses différents rouages. Cette branche du droit pénal pose ainsi les conditions dans lesquelles les infractions sont recherchées, découvertes, constatées, poursuivies et prouvées. Ce domaine concerne directement les autorités compétentes au niveau de leurs actions et de leurs attributions. La procédure pénale précise donc les règles qui président à l'arrestation jusqu'à celles qui motivent la décision de jugement définitif. Elle recouvre ainsi les compétences des différentes juridictions répressives, la recherche et la constatation des infractions, l'administration de la preuve et l'imputabilité de celles-ci aux auteurs présumés. Par conséquent, les règles de procédures pénales établissent les attributions de certains magistrats et représentants de la police judiciaire qui peuvent, dans le respect d'un certain formalisme, porter atteinte aux libertés fondamentales du citoyen. Ainsi, le point d'équilibre entre la volonté d'accomplir la mission de police judiciaire d'une part, et le nécessaire respect des libertés publiques d'autre part constitue le cœur de ce sous-ensemble du droit pénal.

3 Il est néanmoins difficile, aujourd'hui, de parler de " droit pénal européen » ou encore d'« ordre judiciaire européen ». Si la Cour de justice des communautés européennes (CJCE) a été à l'origine de la construction d'un droit communautaire prévalant sur l'ordre juridique national, concernant le droit pénal, les transformations n'ont pas d'incidence directe sur l'ensemble des branches qui le compose, mais ont essentiellement un impact sur les dispositifs facilitant la coopération entre juridictions nationales, appelées aussi " procédures d'entraide judiciaire ». La mise en place de structures comme les magistrats de liaisons, le réseau judiciaire européen ou l'unité Eurojust $^{3} s^{\prime}$ inscrit avant tout dans une logique visant à faciliter l'entraide européenne, et ne répond pas vraiment à une volonté de créer « un ordre pénal européen ». Dans ces conditions, saisir les transformations inhérentes au domaine pénal à l'échelle européenne revient à travailler sur les nouveaux dispositifs mis en œuvre afin de faciliter une plus grande coopération entre les différentes autorités judiciaires nationales au niveau de l'arrestation et/ou de la reconnaissance des jugements.

4 Si la coopération policière à l'échelle européenne s'est développée dès la fin des années 1970, la coopération en matière de justice pénale n'a été instituée qu'au début des années 1990. Cette chronologie ne doit rien au hasard. En effet, il semble que l'une des principales justifications du développement des dispositifs d'entraide judiciaire renvoie à la nécessité de créer " un espace européen de sécurité homogène entre action de police et de justice ${ }^{4}$ ». Face à l'institutionnalisation de plus en plus affirmée de la coopération policière, notamment avec la création d'Europol, nombre d'acteurs judiciaires, administratifs et politiques se sont inquiétés de la mise en place d'une Europe de la police sans système judiciaire complémentaire : "Cela aurait été assez grave du point de vue institutionnel, que la police soit organisée, et pas la justice. Donc pour moi, la coopération au niveau de la justice est essentielle car elle encadre la coopération policière ${ }^{5}$ ». Dans son rapport sur « l'avenir de l'Europe de la justice et de la police " datant de 1999, le Commissariat général du Plan estime de façon concomitante : " [que l'un des besoins essentiels] est celui de la défense des droits individuels. Le déséquilibre entre les progrès de la coopération policière et ceux de la justice est patent. [...] Europol s'est développé sans contrôle juridictionnel ${ }^{6} »$. Au-delà des logiques sociales et politiques qui sous-tendent ces discours, l'introduction d'un cadre judiciaire au sein du 3e pilier de l'Union, qui regroupe depuis le traité de 
Maastricht les questions de "Justice et d'Affaires intérieures ${ }^{7}$ ", constitue un élément fondamental de légitimation pour le développement du domaine européen de la sécurité, en particulier à propos de la question d'une possible force opérationnelle européenne de police judiciaire ${ }^{8}$. L'émergence de dispositifs d'arrestation et d'application des peines à l'échelle européenne s'inscrit donc, de façon plus générale, dans ce processus de développement du domaine de la sécurité européenne.

5 L'objet de cet article est de tracer des pistes d'étude qui permettent de mieux comprendre quelles sont les dynamiques qui sous-tendent la mise en place d'une coopération pénale à l'échelle européenne. Nous partirons de la littérature juridique et institutionnelle déjà importante sur ce sujet, pour expliquer en quoi une approche historique en termes de processus constitue une première piste essentielle pour appréhender les différentes étapes d'une construction qui, loin d'être linéaire, apparaît plutôt comme intermittente et chaotique du fait des luttes institutionnelles et de l'omniprésence de l'agenda politique international et national. Parler de processus implique de saisir ces différentes phases, en pointant les moments de ruptures et de changements dans le souci de dépasser les lectures théoriques préétablies de la construction européenne. La mise en évidence de ces instants critiques conduira, dans un second temps, à placer au cœur de notre analyse les interactions entre les acteurs via l'étude de leurs ressources sociales et de leurs représentations. Enfin, en guise de conclusion nous verrons dans quelle mesure la déconstruction du processus ayant conduit à l'instauration d'instruments pénaux de coopération permet d'interroger la forme institutionnelle et normative que revêt aujourd'hui le régime pénal européen.

Généalogie de la coopération judiciaire européenne

6 Le choix de s'intéresser aux dynamiques sociales à l'origine de la production des règles de coopération pénale en Europe s'inscrit dans une logique de complémentarité par rapport à des approches juridiques qui se focalisent essentiellement sur les questions d'ordre doctrinal. La déconstruction, dans une démarche empirique, du processus qui sous-tend l'avènement d'une coopération pénale européenne, conduit à choisir un cadre méthodologique volontairement exploratoire, afin de ne pas se contenter d'une histoire de la construction européenne de type fédéraliste ou intergouvernemental. Il s'agit, dès lors, d'effectuer une généalogie de ce processus à travers la compréhension de ses principaux chapitres et moments de transformation.

Pour une approche contextuelle des instruments juridiques de l'entraide pénale européenne

7 Les analyses et ouvrages qui prédominent actuellement dans le champ des études sur la coopération pénale européenne s'attachent surtout à décrire les dispositifs de cette coopération et/ou à présenter les futures évolutions jugées nécessaires. La grande majorité de ces études se concentre soit sur les différents dispositifs légaux et politiques de la coopération judiciaire européenne ${ }^{9}$, soit sur les modèles de coopération transnationaux permettant la concordance et/ou l'harmonisation des procédures pénales. La création d'instruments de coopération, comme l'unité de coopération Eurojust et le mandat d'arrêt européen (MAE) ${ }^{10}$, est le plus souvent présentée comme une étape logique et évidente du processus européen en matière pénale. En l'espèce, la définition des principaux dispositifs d'entraide est réalisée à partir de leur contenu, notamment à travers leurs apports et leurs limites. Dès lors, l'analyse s'inscrit généralement dans une logique normative. Le renforcement de la coopération en matière pénale est appréhendé comme le produit dérivé d'une construction 
européenne renvoyant à une histoire inéluctable et " naturelle ${ }^{11}$ ", sans que ne soit posée la question européenne autrement qu'en termes de " progrès » ou de " recul ». La problématique de la souveraineté apparait souvent comme le facteur expliquant les résistances, le statu quo ou les avancées. A partir de ce type d'analyses, il est difficile d'appréhender de façon satisfaisante des questions aussi fondamentales que la temporalité des décisions, les raisons de leur production et leurs conséquences normatives.

D'autres travaux se focalisent sur les formes juridiques qu'un « nouvel espace pénal européen » peut ou doit revêtir. Dans cette perspective, les problématiques s'articulent autour d'une démarche essentiellement juridique et/ou doctrinale. Les questionnements s'intéressent aux principes d'un nouvel ordre pénal européen et aux possibles voies qui permettraient de constituer un ensemble de règles " harmonisées". Relevant de cette dimension doctrinale d'innovation scientifique, les études dont découle le projet Corpus Juris représentent un exemple qui illustre parfaitement la teneur de ce type d'approches ${ }^{12}$. Mêlant à la fois recherche scientifique et prise de position normative dans le débat institutionnel, ce projet interroge le principe d'une territorialité européenne qui, selon les auteurs, est nécessaire " pour les besoins de la recherche, de la poursuite, du jugement et de l'exécution ${ }^{13} »$. En partant de ce concept de territorialité européenne qui regrouperait "l'ensemble des territoires des Etats membres de l'Union en un espace unique ${ }^{14} "$, les juristes proposent, dans le souci de limiter les inégalités de procédure, de créer un Ministère public européen (MPE). Selon eux, ce MPE constituerait une « autorité de la Communauté européenne, responsable pour la recherche, la poursuite, le renvoi en jugement, l'exercice de l'action publique devant la juridiction de jugement et l'exécution des jugements concernant les infractions définies ci-dessus ${ }^{15}$ ». Si les analyses regroupées dans le projet Corpus Juris constituent certainement un exemple particulier, du fait du rôle institutionnel et politique qu'elles revêtent, elles s'inscrivaient néanmoins initialement dans une démarche de recherche juridique, en particulier autour des travaux de Mireille DelmasMarty ${ }^{16}$. Dans le cas de la France, et grâce notamment au soutien des financements européens, il existe un certain nombre de travaux menés dans cette perspective, afin d'élaborer une typologie des modèles juridiques de la coopération judiciaire européenne ${ }^{17}$. Plaçant au cœur de leur raisonnement la question de la normativité, ces dernières analyses sont indispensables à la compréhension de l'architecture légale de la coopération judiciaire européenne, notamment en ce qui concerne la question cruciale de l'équilibre entre répression et liberté publique à cette échelle ${ }^{18}$. Toutefois, étant donné que les systèmes juridiques et leur comparaison constituent l'objet d'étude principal de ces travaux, le raisonnement se contente d'identifier les différences juridiques comme les principales raisons du blocage. Les questions relatives aux acteurs et aux représentations de ces derniers sont souvent ignorées au profit de la doctrine. Le débat européen n'est appréhendé qu'à travers la seule opposition entre les tenants du fédéralisme et les tenants de la souveraineté. La forme que revêt le processus de changement ne constitue pas, en réalité, l'objet de recherche privilégié pour ces études juridiques. Ce type d'approches ne permet donc pas de comprendre l'ensemble des dynamiques qui traversent le droit à l'échelle européenne, notamment celles relatives aux dimensions sociales et politiques structurant la production juridique.

9 Les pistes ouvertes par certaines études consacrées au droit communautaire et à sa construction constituent, sur ce point, des références intéressantes. Les premières analyses concernant la construction du droit communautaire ont porté de manière 
exclusive sur les décisions et la jurisprudence de la Cour de justice des communautés européennes. Toutefois, à partir des années 1990 et de l'arrêt de la Cour constitutionnelle allemande sur le traité de Maastricht, un certain nombre d'auteurs ont insisté sur la nécessité de prendre en considération les dimensions sociales de la construction du droit à l'échelle européenne ${ }^{19}$. Or, le fait de ne pas s'intéresser exclusivement aux problématiques doctrinales permet de poser de nouvelles questions, en particulier en ce qui concerne les mécanismes de production et d'acceptation des nouvelles règles juridiques. Cependant, à la différence des ces études qui restent essentiellement centrées sur les acteurs juridiques, il semble nécessaire, en particulier dans le domaine pénal, de ne pas exclure la dimension politique que peuvent revêtir certaines mobilisations juridiques. Ainsi, au-delà d'une simple description des décisions et dispositifs européens en matière de coopération pénale, il est particulièrement utile de réaliser un véritable travail de déconstruction du processus de production, en s'interrogeant sur ses dynamiques constitutives, qu'elles soient d'ordre juridique, social et politique.

10 L'étude de la construction de règles pénales transnationales permet, à l'instar des travaux de Jacques Commaille, d'envisager le droit comme un produit social impliquant une analyse des catégories issues du sens commun, ainsi que des pratiques et représentations des acteurs, des mécanismes de traduction et d'interprétation de la norme ${ }^{20}$. Saisir les dynamiques constitutives de ces instruments, qui sont des produits de l'action publique, conduit, selon Pierre Lascoumes et Patrick Le Galès, à " matérialiser et à opérationnaliser l'action gouvernementale ${ }^{21}$ ". Suivant ce raisonnement, " il s'agit non seulement de comprendre les raisons qui poussent à retenir tel instrument plutôt que tel autre, mais d'envisager également les effets produits par ces choix ${ }^{22}$ ». Les outils ne sont donc pas neutres, mais porteurs de normes et de représentations du social. L'étude des différents instruments judiciaires institués pour favoriser ou renforcer la coopération judiciaire illustre également les dimensions techniques et politiques à l'œuvre dans la réalisation de la coopération pénale européenne. La politisation à l'extrême de certains outils (comme Eurojust et le MAE) ou, à l'inverse, la grande technicité qui entoure certains dispositifs (comme l'échange d'informations et le principe de reconnaissance mutuelle des décisions pénales) - voire la mise à l'écart d'autres domaines (le Procureur européen, le droit procédural de la défense) - doivent être considérées à l'aune des enjeux qui structurent les luttes entre les bureaucraties européennes et nationales. L'imposition de certaines règles institutionnelles et juridiques se réalise au détriment d'autres. Néanmoins, la fluidité des croyances et du jeu politique et institutionnel fait parfois revenir certains projets écartés sur le devant de la scène. La mobilisation d'une approche en termes de processus a alors l'avantage de pouvoir retracer une généalogie de la coopération judiciaire européenne à travers ses dynamiques et les principaux moments de mutations.

L'européanisation du domaine judiciaire

11 Cette démarche, qui insiste sur les processus, a des implications fortes au niveau méthodologique. En effet, il s'agit de restituer la genèse et l'institutionnalisation d'un domaine européen à travers l'étude de ses principales séquences, mais aussi et surtout via l'identification des périodes de changements qui permettent de comprendre les mécanismes de passage d'une phase à une autre. L'idée de préconiser une approche de la construction européenne en termes de processus n'est certes pas nouvelle. Les approches néo-fonctionnalistes ont placé au cœur de leur raisonnement ce postulat à 
travers le concept de "spill over ». Selon celui-ci, la croissance des échanges transnationaux dans un secteur donné crée une pression sur les règles nationales disparates qui, progressivement, vont être remplacées - via des ajustements et une institutionnalisation grandissante - par des règles supranationales renforçant la gouvernance communautaire ${ }^{23}$. Au regard des éléments empiriques, il est indéniable que le processus européen à l'œuvre dans le domaine de la coopération pénale ne correspond pas à un transfert progressif et inéluctable de compétences du national vers le supranational. La " renationalisation ", notamment dans la mise en œuvre des instruments judiciaires de coopération, constitue un phénomène largement constaté. Dans le cas du MAE, on assiste ainsi à ce qu'on pourrait appelait une " double renationalisation » : d'une part au niveau des chancelleries des Etats membres - dans leur transposition de la décision-cadre relative à la création du MAE - et, d'autre part, à l'échelon des Cours constitutionnelles nationales ${ }^{24}$, dans la mise en œuvre juridique de ce dispositif. Concernant l'unité Eurojust, cette renationalisation s'inscrit, comme nous le préciserons par la suite, dans la logique intergouvernementale de cette structure avec une entière liberté laissée à chaque Etat quant à la définition du statut et des pouvoirs juridiques dévolus à leurs représentants. Dans le cas d'un secteur dominé par des règles institutionnelles intergouvernementales, comme celui de la coopération pénale, c'est-à-dire où les Etats restent les principaux décideurs, l'idée de processus européen au sens néo-fonctionnaliste constitue une piste trop limitée, qui ne saurait prendre en compte les différentes évolutions observées.

La notion d'européanisation offre un cadre plus adapté sur cette problématique, comme le montre la définition qu'en donne Claudio Radaelli :

«L'européanisation fait référence à : un processus de (a) construction, (b) de diffusion et (c) d'institutionnalisation de règles formelles et informelles, de procédures, de paradigmes politiques, de styles, de "manière de faire", de croyances et normes qui sont dans un premier temps définis et consolidés dans la production politique de l'Union européenne, puis intégré dans les logiques nationales au niveau des discours, des identités, des cadres politiques et des politiques publiques ${ }^{25}$ ».

13 L'un des avantages de cette définition est, à l'inverse des approches néofonctionnalistes, de considérer l'européanisation comme un processus à expliquer et non comme un résultat. Dans un premier temps, cette notion d'européanisation a été utilisée dans les études portant sur l'impact national des politiques européennes ${ }^{26}$. L'objet de ces recherches était de mettre en évidence, selon les secteurs, les conséquences de la construction européenne sur les politiques et les systèmes institutionnels nationaux. Ces premiers travaux ont permis de mieux appréhender une partie des dynamiques européennes. Cependant, dans le domaine de la coopération judiciaire il est nécessaire de ne pas limiter la construction aux simples effets " domestiques » de l'Europe car il convient de se référer au processus dans sa globalité, incluant la formulation des politiques européennes, leur mise en œuvre à l'échelle nationale et les effets de feed back du niveau national vers le niveau européen ${ }^{27}$. L'évolution et la place d'une unité communautaire telle que l'office de lutte anti-fraude (OLAF) ${ }^{28}$ montre combien il est crucial de mobiliser le niveau communautaire dans l'étude de la transformation des dispositifs de coopération pénale. Le fait d'élargir l'analyse au-delà du seul impact national permet d'évaluer précisément l'influence que les luttes au sein des institutions communautaires ont sur la forme et la vitesse d'institutionnalisation de la coopération judiciaire européenne. Si l'européanisation ne 
se produit pas seulement au niveau des résultats, il faut alors considérer l'ensemble des moments du processus.

Une telle démarche présente l'avantage de dépasser l'opposition, souvent stérile, qui structure les théories néo-fonctionnalistes et intergouvernementales à l'origine des premiers travaux sur l'Union européenne ${ }^{29}$. Le domaine de la coopération judiciaire constitue un exemple parfait de la porosité existant entre ces deux logiques d'analyse. Loin d'être " concurrentes ", ces visions de la construction européenne semblent, en réalité, se valider lors des différentes phases du processus d'institutionnalisation de la coopération pénale. On assiste à une alternance entre des dynamiques de "spill over " et des logiques intergouvernementales dans lesquelles les gouvernements réaffirment leur autorité décisionnelle. La fluctuation entre ces deux mouvements structure d'ailleurs l'ensemble du processus, chacun prenant le pas sur l'autre en fonction du contexte politique et institutionnel. Cette relative complémentarité doit être comprise à travers l'idée que ces deux approches n'étudient pas le même objet et s'intéressent à des moments différents $d u$ changement institutionnel. L'approche en termes d'européanisation a le mérite de travailler de façon plus inclusive et exploratoire, dans le souci de ne pas vouloir à tout prix donner une lecture théorique de l'Union européenne, sans jamais interroger la réalité des formes qu'elle revêt. La méthode empirique, correspondant à cette acceptation de l'européanisation comme " processus d'ajustements institutionnels, stratégiques et normatifs induits par la construction européenne ${ }^{30}$ ", consiste dans un premier temps à raconter une histoire, en identifiant ses principaux chapitres.

Genèse de la coopération judiciaire européenne.

Dès 1957, l'entraide en matière répressive donne lieu à la conclusion d'une Convention européenne d'extradition. Elle sera suivie par la Convention européenne d'entraide judiciaire du 20 avril 1959. Il faut attendre la fin des années 1970, avec l'émergence de la question de la lutte contre le terrorisme, pour que de nouveaux textes soient élaborés en faveur d'une plus grande coopération pénale entre les Etats membres. On assiste alors à une multiplication de projets concurrents qui, pour la plupart, ne verront jamais le jour du fait des oppositions entre les Etats. Chargé d'élaborer une convention par laquelle ces derniers s'engagent à arrêter et à extrader les auteurs de prises d'otages, le premier groupe de travail ad hoc est pris de vitesse par le Conseil de l'Europe. Ce dernier, sur proposition belge, présente, le 27 janvier 1977, une Convention pour la répression du terrorisme ${ }^{31}$. Cependant, plusieurs Etats refusent de ratifier cet acte, en raison, officiellement, de la trop grande hétérogénéité des systèmes judiciaires ${ }^{32}$.

16 Ce blocage conduit à l'accord de Dublin en date du 4 décembre 1979 : celui-ci prévoit l'application de la Convention du Conseil de l'Europe uniquement entre les Etats membres des Communautés européennes, en obligeant tous les signataires à poursuivre et à extrader les auteurs d'actes terroristes. Si la France accepte de signer ce texte, elle le fait en contrepartie d'une avancée sur son projet de Convention de coopération en matière pénale. Après plus de deux ans de travail et une signature prévue lors de la conférence des ministres de la Justice des neuf Etats membres de la communauté (le 19 juin 1980 à Rome), ce premier projet de coopération judiciaire à l'échelle européenne est finalement rejeté, du fait du refus des Pays-Bas, soucieux de privilégier le cadre du Conseil de l'Europe. 
La relance de la coopération judiciaire : les accords de Schengen et le traité de Maastricht.

17 A la suite de ces premières tentatives avortées, la question de la coopération judiciaire ne sera véritablement relancée que dix ans plus tard, avec les accords de Schengen signés le 14 juin 1985 et complétés par une convention d'application du 19 juin 1990. Si l'objectif le plus «significatif » de ces accords est de supprimer graduellement tout contrôle aux frontières intérieures afin de faciliter la circulation des personnes, il s'accompagne d'un dispositif prévoyant le renforcement de la coopération judiciaire au niveau de l'extradition et de la transmission des jugements répressifs ${ }^{33}$.

En 1992, le traité de Maastricht, en créant le « 3e pilier » de l'Union européenne consacré exclusivement aux questions de "Justice et Affaires intérieures ", met en place, dans un nouveau domaine, les contours d'un système intergouvernemental qui revêt des caractéristiques spécifiques. D'une part, toutes les décisions sont prises à l'unanimité par les Etats membres. D'autre part, le fait que les Etats soient les principaux décideurs se traduit par l'absence d'initiatives de la Commission. Enfin, il n'existe ni sanction ni instrument coercitif en cas de non-respect des engagements, ce qui permet donc à chaque Etat de suivre sa seule volonté. Les orientations du traité de Maastricht se concrétisent dans le domaine judiciaire à partir de 1993, par la mise en place des premiers échanges de magistrats entre pays membres de l'Union. Mis en avant par les autorités françaises dans le cadre de ses relations avec l'Italie et les PaysBas, le système des magistrats de liaison, dont le modèle est largement influencé par celui des officiers de liaison de la coopération policière, a pour objectif de faciliter la coopération judiciaire au niveau européen dans une logique bilatérale entre chaque Etat membre ${ }^{34}$. Comme le soulignent la plupart des magistrats actuellement en poste, au-delà de cette mission d'entraide, ce dispositif joue également un rôle important dans les relations diplomatiques entre Etats, notamment dans le cas d'affaires judiciaires jugés délicates. Ce système a été érigé en dispositif européen, sorte de «bonne pratique européenne » par une action commune du Conseil européen du 22 avril $1996^{35}$.

Le traité d'Amsterdam et la création de l'unité Eurojust.

Le traité d'Amsterdam de 1997 représente une étape fondamentale dans l'évolution " du 3e pilier " de l'Union européenne au niveau de l'architecture institutionnelle et juridique ${ }^{36}$. C'est à partir de cette base juridique que sera initié deux ans plus tard le programme de Tampere, via l'instauration d'une action commune. Cette action a pour but de faciliter et d'accélérer la coopération entre les Etats membres au niveau des procédures (y compris dans le domaine de l'extradition). Elle vise aussi à faire adopter progressivement des mesures instaurant des règles minimales relatives aux éléments constitutifs des infractions pénales et des sanctions applicables ${ }^{37}$. Le 29 juin 1998, le Conseil européen adopte à Birmingham une autre action commune stipulant la création d'un Réseau judiciaire européen dont l'objectif est de favoriser l'échange d'informations entre les Etats membres en matière judiciaire ${ }^{38}$. La mise en place d'une telle structure s'inscrit une fois de plus dans une approche bilatérale privilégiant les contacts informels et temporaires ${ }^{39}$.

20 S'appuyant sur cette orientation institutionnelle et désireux, à l'instar des autres pays, de marquer son temps de présidence par des décisions politiquement et médiatiquement importantes, le gouvernement finlandais décide d'organiser, le 15 et 16 octobre 1999 à Tampere, le premier sommet européen consacré exclusivement aux questions de sécurité dans l'Union. Cette réunion a pour objectif déclaré « la création 
d'un espace de liberté, de sécurité et de justice dans l'Union européenne ${ }^{40}$ ». Les termes exacts utilisés dans le traité d'Amsterdam ${ }^{41}$ sont ainsi repris. C'est alors que le Conseil européen décide :

« afin de lutter contre les formes graves de criminalité organisée [...] la création d'une unité (Eurojust) composée de procureurs, de magistrats ou d'officiers de police ayant des compétences équivalentes, détachées par chaque Etat membre conformément à son système juridique. Eurojust aura pour mission de contribuer à une bonne coordination entre les autorités nationales chargées des poursuites et d'apporter son concours dans les enquêtes relatives aux affaires de criminalité organisée $[. . .]^{42}$ ».

21 Eurojust constitue ainsi la première unité judiciaire européenne multilatérale, marquant une véritable évolution institutionnelle par rapport aux systèmes des magistrats de liaison et du Réseau judiciaire européen. Suite à cette décision, les institutions du Conseil européen et certains Etats à l'origine du projet (France, Belgique, Portugal et Suède) vont avancer l'idée de mettre en place un Pro-Eurojust provisoire avant de créer l'unité définitive. Du fait du peu d'engagement institutionnel qu'engendre cette proposition transitoire, Pro-Eurojust est adopté par les Etats au Conseil JAI du 14 décembre 2000 lors du sommet de Nice. Seule la Commission européenne s'est prononcée contre cette unité transitoire, position relayée au niveau du Parlement européen. En décembre 2001, le Conseil européen de Laeken confirme que l'unité Eurojust peut commencer à développer ses activités dans ses locaux de La Haye. Le sommet de Tampere a également été à l'origine d'une ouverture juridique et institutionnelle qui a abouti à la construction d'un projet de procédure d'extradition entre les Etats membres.

Le mandat d'arrêt européen.

Durant le sommet de Tampere, les questions relatives à l'extradition ont également été largement abordées, du fait notamment de l'activité des membres du Secrétariat général du Conseil (SGCE). Devant les réticences importantes d'une grande majorité des membres du Conseil européen, le Secrétariat général du Conseil va exploiter le contexte politique particulier de l'époque comme le raconte un de ses principaux représentants :

« Je raconte souvent que c'était dans un contexte assez curieux. Au moment de la relecture des conclusions de Tampere, le Premier ministre italien de l'époque, Giuliano Amato, a dit quelque chose que personne n'a vraiment compris. Il a parlé de Schengen, de l'extradition ... A la suite de sa déclaration il nous a fourni une partie de son commentaire. Il fallait aller très vite car le deuxième jour, les conclusions sortent devant les journalistes. La mise en forme du texte se fait très souvent dans l'urgence et nous n'avons pas toujours le temps de bien approfondir le texte. Nous avons pris le texte d'Amato qui paraissait avoir du sens, et on l'a collé sous le paragraphe concernant la suppression de la procédure d'extradition avec comme phrase de conclusion, "le Conseil invite la Commission à travailler sur l'extradition ...”. Le lendemain de Tampere puisque nous n'avions plus le temps de revoir le texte, cela voulait dire que le Conseil européen demandait à la Commission de faire une proposition sur la procédure d'extradition ${ }^{43} »$.

A l'époque, l'Italie se trouvait dans un contexte politique difficile avec la question de l'extradition du chef du PKK, Abdoulla Oçalan. En raison d'un signalement introduit par les autorités allemandes dans le système Schengen SIS, la police italienne l'avait arrêté de façon fortuite lors d'un contrôle d'identité, et devait l'extrader vers l'Allemagne. Cependant, en raison de la sensibilité politique de ce dossier, au niveau intérieur et 
extérieur, les autorités allemandes avaient préféré laisser le détenu encombrant à la justice italienne. Ainsi comme l'explique le représentant du SGCE :

« en fait ce que d'Amato voulait, c'était de demander à la Commission de travailler sur le volet SIS lié à l'extradition. Il voulait que si un pays émet une demande d'extradition, il s'engage à la suivre quelle que soit la situation que cela entraîne. On a utilisé cette occasion pour faire avancer cette question sur laquelle tout était bloqué ${ }^{44} »$.

Se saisissant de cette opportunité ouverte par les conclusions de Tampere ${ }^{45}$, la Commission va mettre en place un processus classique d'expertise afin de construire un projet de procédure d'extradition simplifiée entre les Etats membres. Initialement prévu pour être présenté fin septembre 2001 par la Commission, le projet de MAE va connaître une adoption un peu particulière du fait, là encore, du contexte politique ${ }^{46}$.

Quelques jours après les attentats du 11 septembre 2001, les chefs d'Etats et de gouvernements de l'Union européenne, réunis en sommet extraordinaire à Bruxelles, décident d'adopter le projet de MAE avant la fin 2001. Produit d'une décision hautement politique et du souci de prouver à la fois aux Etats-Unis et aux opinions européennes que l'Union européenne et ses membres sont décidés à lutter activement contre le terrorisme grâce à une coopération accrue, le MAE est politiquement entériné dans un délai record, les 6 et 7 décembre 2001, par le Conseil JAI au sommet de Laeken. Un membre de la Commission souligne que « les événements du 11 septembre n'ont pas accéléré les choses du point de vue de la commission puisqu'au lieu du 28 ou du 29 septembre, c'est le 19 septembre que le projet a été adopté par la Commission ${ }^{47}$ ». Il semble toutefois que les attentats aient influencé la temporalité et le contenu de la décision des Etats, principaux décideurs du processus : " on a même eu du mal à comprendre ce que le Conseil nous demandait, parce qu'une négociation pareille en trois mois paraissait totalement impossible ${ }^{48} \gg$. Finalement, c'est le 13 juin 2002 que la décision-cadre relative au mandat d'arrêt et aux procédures de remise entre les Etats sera adoptée officiellement, en vue d'une entrée en vigueur du nouveau système d'extradition à compter du 1er janvier 2004, dans l'ensemble des pays membres. Ce nouvel instrument juridique de coopération marque officiellement la fin d'une pratique politique et administrative de l'extradition au profit d'une procédure uniquement judiciaire, ayant pour objectif affiché un raccourcissement des délais. Selon la décisioncadre du 13 juin 2002, le dispositif du MAE devait être opérationnel à partir du 1er janvier 2004. Or, début 2004, huit pays membres ne l'avaient toujours pas introduit dans leur législation : l'Allemagne, la Grèce, l'Italie, la Lettonie, Malte, la Slovaquie et la République tchèque. En outre, certains Etats, pour des raisons politiques ou juridiques, ont remis en cause leurs engagements pris lors du sommet de Laeken, comme la Pologne, sur la question du pouvoir d'extrader ses propres nationaux. Comme le souligne un membre de la Commission : « maintenant que l'on est en train de regarder les législations de transposition, on voit que le pouvoir politique qui est sorti par la porte a tendance à vouloir rentrer par la fenêtre ${ }^{49}$ ". Si aujourd'hui le MAE est largement utilisé par les autorités judiciaires, il n'en demeure pas moins que son cadre juridique et normatif reste encore très instable dans sa mise en œuvre, à la fois au niveau institutionnel et juridique ${ }^{50}$. Durant le second semestre 2004, la présidence néerlandaise présente le programme de La Haye qui, tout en tirant le bilan du programme de Tampere, relance officiellement un nouveau plan pluriannuel de construction de la coopération judiciaire jusqu'en $2010^{51}$. 
26 Après avoir identifié les principales phases de l'européanisation de la coopération pénale, il convient d'interroger directement les dynamiques de changements qui soustendent cette histoire à la fois en termes institutionnels, normatifs et sociaux. Il s'agit ainsi de répondre aux questions de la temporalité et de la forme des dispositifs et règles judiciaires mis en œuvre.

La coopération pénale européenne saisie par les interactions sociales

27 Comprendre les phases de transformation qui structurent le phénomène d'européanisation de la coopération pénale conduit à travailler sur les interactions qui se jouent entre les acteurs et les différents niveaux de pouvoir. Grâce à la mobilisation des instruments d'analyse de l'action collective, il est possible d'appréhender les problématiques classiques de mobilisations et de constructions d'identités collectives à travers la prise en compte des logiques de compétitions et de résistances. Cela permet de mieux appréhender les structures d'opportunité politique qui façonnent parallèlement la production des dispositifs d'entraide judiciaire.

28 En relayant les pistes ouvertes ici même par les approches de sociologie politique du processus européen ${ }^{52}$, notre travail consiste à placer les acteurs au cœur de l'étude, qu'ils soient individuels ou collectifs. Il s'agit de comprendre comment les jeux institutionnels et sociaux influent sur les comportements des acteurs et jusqu'où ils pèsent sur leurs décisions et leurs représentations. Une telle approche implique de travailler à la fois sur les configurations globales du jeu institutionnel mais aussi sur les parcours, les mécanismes d'adhésion et de socialisation de ces acteurs dont les stratégies ont un impact direct sur la forme que revêt la coopération judiciaire européenne. Dans quelles conditions des acteurs, qui, pourtant, évoluent dans des cadres d'action hétérogènes (fonctionnaires européens et nationaux, magistrats, membres d'ONG, policiers, avocats, universitaires), se réunissent-ils au sein de l'espace européen? Quels sont les acteurs prédominants et comment s'imposent-ils par rapport aux autres, notamment vis-à-vis de ceux qui sont relégués à la marge ?

La compréhension des logiques d'action des différents acteurs réunis dans la construction de la coopération européenne nécessite certes de s'intéresser à leurs ressources et à leurs rationalités, mais aussi d'interroger leurs représentations. Comme le soulignent Christian Lesquene et Andy Smith, un certain nombre d'études " interdépendantistes " des politiques européennes " occulte les phénomènes de représentation et de symbolique politiques. [...] L'analyse tend à privilégier les contacts observables entre acteurs institutionnels mais néglige les représentations sociales qui portent ces mêmes acteurs ${ }^{53}$ ». En écho à une telle critique et dans la perspective de ce que représente selon nous la notion d'européanisation, le choix d'articuler les hypothèses explicatives à partir de la matrice de variables - idées, intérêts et institutions - s'impose comme un postulat important ${ }^{54}$. Il s'agit encore une fois de proposer une méthode empirique dont l'objet est avant tout de déconstruire les mécanismes à l'œuvre plutôt que de tenter de les classifier selon une lecture préétablie. En effet, rien ne dit que les mêmes types de variables ont le même poids explicatif pour chaque phase d'européanisation de la coopération judiciaire. On comprend aisément que la représentation de la «menace transnationale terroriste ", ne revêt pas la même importance lors du Conseil de Tampere en 1999, dont les conclusions créent l'unité Eurojust, et lors du sommet de Laeken en décembre 2001 durant lequel est accepté politiquement le projet de MAE. A partir de la compréhension des logiques sociales et institutionnelles qui sous-tendent la coopération pénale européenne, il est donc 
indispensable de poser la question des représentations à la fois à travers l'évolution des croyances et des cadres de pensée qui s'imposent, et via la façon dont ceux-ci sont " travaillés " par les différents acteurs. Comment les problèmes et les enjeux qui structurent et légitiment cette européanisation du judiciaire se construisent-ils ? Dans quelle mesure les représentations tendent-elles à s'autonomiser et à peser sur les stratégies des acteurs ? L'explication à partir des trois variables idées, intérêts et institutions ne saurait être pertinente que si on la complète par la variable politique. Dans l'histoire de l'européanisation de la coopération pénale, certains grands moments de changements doivent être appréciés à l'aune de « fenêtres d'opportunité politiques ", comme ce fut le cas pour le MAE avec le 11 septembre 2001.

Le champ de la coopération pénale européenne.

30 Comme nous l'avons souligné en définissant l'européanisation, travailler sur les interactions sociales qui structurent ce processus dans le domaine de la coopération judiciaire implique de considérer la relation entre le niveau européen et le niveau national à la fois de façon " descendante » et « ascendante ». Cela permet en réalité de considérer les différents types d'interactions et de saisir « la diversité des intérêts en présence et les rapports qui se nouent à différents niveaux entre les acteurs intéressés 55 ». Dès lors, à la différence de l'approche intergouvernementaliste où l'interaction principalement étudiée est celle qui existe entre les Etats au moment de la négociation, l'européanisation nous conduit à interroger les dynamiques relationnelles entre l'ensemble des acteurs. En termes de méthode, cela signifie qu'il faut identifier les acteurs entre qui se jouent les interactions afin de caractériser ces relations et de comprendre leur évolution.

31 La mobilisation du concept de champ, élaboré par Pierre Bourdieu et reprise par un grand nombre de chercheurs, présente l'avantage de pouvoir considérer ces différentes dimensions, par l'analyse de l'ensemble des acteurs dans un même espace social à travers leurs prises de position et leurs interactions ${ }^{56}$. Dans sa transposition du concept de champ au domaine de la sécurité, Didier Bigo souligne, en s'appuyant sur les hypothèses de Peter Katzenstein, la nécessité " de dépasser les lignes habituelles tracées par les sciences sociales entre externe et interne, entre problème de défense et de police, entre problème de sécurité nationale et problème d'ordre public ${ }^{57}$ ». Dans le cas de l'européanisation de la coopération judiciaire, cela permet, d'une part, de travailler sur les acteurs nationaux et européens dans une même configuration à plusieurs niveaux et, d'autre part, d'insérer la problématique de la coopération judiciaire, en tant que sous-champ, dans celle, plus large, de la construction d'un champ de la sécurité à l'échelle transnationale. La théorie du champ ouvre ainsi la possibilité d'appréhender l'ensemble des acteurs de la coopération judiciaire européenne et pas seulement ceux qui, considérés comme dominants, appartiennent au triangle institutionnel européen (Etats, Conseil européen et Commission européenne). L'intérêt d'utiliser un tel concept est alors de pouvoir saisir les dynamiques intersectorielles et de montrer la façon dont certains agents se sont imposés dans le champ de la sécurité européenne alors que d'autres ont été confinés ou relégués au statut d'" outsiders » ou d'« amateurs ». L'étude du champ pénal européen consiste à définir les écarts de positionnement entre les acteurs et à appréhender les effets que ceux-ci ont sur les prises de positions et les logiques discursives ${ }^{58}$. Les jeux politiques et institutionnels dans lesquels les agents interviennent doivent être pensés dans une démarche dynamique à partir des changements des lignes d'affrontements et des positions. En fonction du moment du processus d'européanisation, certains acteurs 
peuvent jouer un rôle plus ou moins important ${ }^{59}$. En mettant en lumière les objectifs, les ressources et les préférences des acteurs, il s'agit de recomposer concrètement les interactions entre les institutions européennes, les gouvernements nationaux et les acteurs sectoriels dans le champ de la coopération pénale européenne.

On assiste à un processus à plusieurs niveaux ${ }^{60}$ et plusieurs phases, traversé par des dynamiques de compétition / réaction / collaboration entre les principaux acteurs que sont les Etats, le Conseil européen, la Commission européenne et les entrepreneurs ${ }^{61} \mathrm{du}$ droit. Soucieuse de s'impliquer davantage dans le pilier "Justice, liberté et sécurité " (JLS) où ses compétences sont très limitées, la Commission propose des initiatives que les Etats subissent et/ou bloquent, dans une logique de compétition. Si la Commission cherche avant tout à étendre ses prérogatives, les chancelleries nationales gardent leur pouvoir de décision. Décidée à Tampere par le Conseil européen en 1999, la construction de l'unité Eurojust doit se comprendre, pour une part, comme la réponse des Etats aux ambitieux projets communautaires d'intégration dans le domaine pénal qui ont provoqué « des réactions extrêmement vives, dans les Etats membres, chez les politiques et les chancelleries ${ }^{62} 》$. En instituant une unité de coopération judiciaire aux logiques purement intergouvernementales, les Etats ont voulu conserver leur pouvoir de décision et d'orientation sans remettre en cause l'européanisation du domaine judiciaire, notamment en raison de contextes politiques intérieurs particuliers. Les autorités étatiques qui apparaissent comme les principaux acteurs dans le domaine intergouvernemental de la coopération judiciaire ne sont en aucun cas homogènes. Les positions qu'ils défendent dans les discussions diplomatiques sont le fruit, là aussi, de jeux nationaux complexes et hétérogènes qui fluctuent en fonction des pays. A ce titre, la place que peut occuper le judiciaire dans la configuration et l'équilibre de chaque système politique et institutionnel national, notamment vis-à-vis de la police, est un facteur important. De manière classique, on constate que les formes d'organisation nationale ont un impact direct sur les négociations européennes et sur leurs orientations ${ }^{63}$. Dans le cas du champ de la coopération pénale européenne, la structure juridique intergouvernementale du troisième pilier fait du Conseil européen un lieu essentiel dans la construction des politiques de coopération ${ }^{64}$. Comme ont pu le démontrer les études sur les working groups et les groupes ad hoc du Conseil européen, les négociations européennes mettent en scène un certain nombre d'acteurs intermédiaires qui se situent au croisement des jeux juridiques nationaux et des processus transnationaux ${ }^{65}$. C'est, par exemple, le cas du Secrétariat général du Conseil qui occupe une place importante dans le processus décisionnel de la coopération judiciaire ${ }^{66}$. Pour le MAE, si la Commission a été à l'origine de la proposition validée politiquement à la suite du 11 septembre, la décision-cadre finale du 13 juin 2002 a fait l'objet d'une réécriture conséquente par la présidence belge, en étroite collaboration avec certains membres du SGCE.

Parallèlement à ces dynamiques de compétition, les relations interinstitutionnelles peuvent également s'inscrire dans des logiques de collaboration. La Commission devient un pourvoyeur de diagnostics, de solutions et d'agendas face à des Etats qui ne cessent de réaffirmer leur autorité décisionnelle (comme dans le cas du MAE ${ }^{67}$ ). En transformant son mode de participation au processus sans remettre en cause ses orientations ${ }^{68}$, la Commission réinvestit le terrain en tant que force de proposition. Cette relation de collaboration ne s'opère pas dans une logique de concession, dans la mesure où les acteurs continuent à travailler chacun dans leur intérêt, comme cela a été le cas pour le MAE. La Commission a besoin des gouvernements pour donner les 
impulsions dans des secteurs où ses compétences sont limitées, et les gouvernements ont besoin de la Commission pour produire des instruments de mise en œuvre, de financement et d'évaluation des projets. Les conclusions de Tampere marquent l'institutionnalisation de ce registre de participation ${ }^{69}$, ce qui a pour effet de renforcer la volonté de la Commission de s'investir dans ce domaine "Justice, liberté et sécurité " afin de constituer un pôle efficace de proposition face aux Etats. Après une intense concurrence au sein même de la Commission entre les différentes Directions générales ${ }^{70}$, la DG « Justice, liberté et sécurité » s'est progressivement autonomisée, au point de constituer aujourd'hui un pôle auquel se rattache un corpus d'analyses et de programmes de plus en plus dense en faveur d'un renforcement de l'intégration judiciaire. Connaissant un accroissement continu de ses domaines de compétences et de ses ressources humaines, cette enceinte est devenue un lieu de spécialisation pour un certain nombre de praticiens nationaux. L'accroissement affirmé de la DG « Justice, liberté et sécurité » a eu pour conséquence de transformer l'Union européenne en " niveau de ressources » dans le domaine de la coopération judiciaire, en particulier par un travail de socialisation et de financement communautaire ${ }^{71}$. Si les intérêts de la Commission et des professionnels s'orientent en faveur d'un renforcement de la coopération, cela ne signifie pas que ces acteurs ont les mêmes objectifs et les mêmes motivations. Chacun constitue pour l'autre une ressource stratégique importante, en termes de légitimité, de moyens et de connaissances. Les professionnels perçoivent l'Europe soit comme un moyen d'influencer le processus de coopération selon des préférences et des intérêts sectoriels, soit dans une stratégie plus individuelle de carrière et de spécialisation. Cette constatation empirique renvoie directement aux processus de professionnalisation à l'échelle européenne.

Il est difficile de réellement comprendre les logiques du champ de la coopération pénale européenne sans considérer deux phénomènes centraux : d'une part, l'investissement au cœur du champ d'un certain nombre de professionnels de la justice et, d'autre part, les acteurs qui restent à la marge du fait des logiques institutionnelles et des coûts d'entrée et d'investissement dans le champ.

Pourquoi et comment se constitue une expertise de la coopération judiciaire européenne à travers la spécialisation d'acteurs nationaux qui, pour certains, deviennent de véritables entrepreneurs de la coopération pénale ? Pour Didier Georgakakis : "Analyser le processus de spécialisation des agents dont une partie de l'activité est en relation avec les institutions européennes, c'est, du même coup, s'interroger plus largement sur la différenciation d'un nouvel espace d'activité politique ${ }^{72}$ ». Cependant, ce politiste souligne que " l'intégration européenne ne passe pas seulement par la formation d'un corps de professionnels "vivant de et pour" les institutions européennes ${ }^{73}$ ». Le cadre national demeure une dimension essentielle dans le phénomène d'européanisation des carrières, en particulier dans un domaine intergouvernemental comme la coopération judiciaire. Il est alors indispensable de se demander pourquoi des magistrats se sont investis concrètement dans la coopération judiciaire, alors même que la majorité des juges reste encore réticente. Quels sont les professionnels du judiciaire qui s'engagent dans le champ de la coopération pénale européenne et selon quelles modalités ? Ont-ils tous les mêmes ressources et les mêmes stratégies? Quelles sont les résistances dans le secteur professionnel de la magistrature ? Comment se construit une nouvelle expertise judiciaire et quels sont les processus de légitimation de ce nouveau modèle ? Les professionnels que sont les entrepreneurs politiques et les détenteurs d'expertise ne constituent pas un groupe homogène. Ils 
interviennent aux différents niveaux de la coopération européenne, soit à travers des mobilisations professionnelles dans lesquelles ils se positionnent comme représentants d'un secteur socioprofessionnel ${ }^{74}$, soit à travers une participation au niveau institutionnel, à l'échelle nationale et européenne (chancelleries, Représentations permanentes, institutions communautaires, juridictions nationales), ou encore en tant que praticiens du judiciaire. Le fait de participer à l'une de ces mobilisations ne signifie pas que ces acteurs occupent des places fixes. En effet, on constate une circulation importante de certains acteurs du fait de leur multipositionnement institutionnel.

Certains magistrats nationaux jouent un rôle important d'entrepreneurs politiques et de détenteurs d'expertise auprès des institutions communautaires ou nationales. Ils ont, en effet, investi ces institutions et participent à la rédaction des projets au sein de la Direction générale "Justice, liberté et sécurité ", des chancelleries des Etatsmembres ou des groupes de travail européens. Ces magistrats doivent être considérés comme les entrepreneurs d'une nouvelle expertise judiciaire, tant au niveau institutionnel, que juridique et moral ${ }^{75}$. Ainsi, interroger directement cette catégorie d'acteurs et les logiques sociales qui prévalent au sein des groupes d'expertise dans le domaine de la coopération judiciaire, et plus largement dans celui de la sécurité, permet de prendre en compte une part importante des modes de production des normes ${ }^{76}$. Il est, par exemple, courant que des magistrats occupant un poste dans leur ministère national en lien avec les questions européennes, soient nommés au niveau européen en tant que représentant national ou au sein même d'une unité européenne (SGCE, OLAF, Eurojust, Représentation permanente, magistrats de liaison). D'autres magistrats peuvent revenir du niveau européen afin de prendre de nouveau un poste à l'échelon national, n'ayant aucune relation directe avec la coopération judiciaire. Cependant, dans ce cas, ils restent très proches des questions européennes, à travers de nombreuses initiatives - valorisation de leur expérience - et constituent de véritables entrepreneurs de la coopération européenne auprès des autres juges qu'ils côtoient.

Les magistrats occupent également une place dans le champ pénal européen comme détenteurs du " pouvoir matériel ». Les travaux de sociologie du droit nous permettent d'apprécier l'importance des magistrats dans la construction et l'évolution de l'objet juridique. De façon générale, il est admis que si l'usage symbolique de ce dernier relève du privilège des gouvernants et des législateurs, les magistrats sont en grande partie maitres de la technique juridique en tant qu'opérateurs. Cette dimension se retrouve dans les dispositifs de coopération comme Eurojust et les magistrats de liaisons. De l'aveu même de leurs représentants, ces instruments ne peuvent se pérenniser et donner des résultats que si les magistrats nationaux s'en saisissent et y recourent. La question est alors de savoir comment et pourquoi les magistrats nationaux vont investir ces moyens de coopération. Les prises de position de ces professionnels du niveau national varient alors entre apprentissage et résistance. De nos entretiens effectués avec des magistrats français, belges, néerlandais et italiens, il résulte que la majorité d'entre eux restent, pour différentes raisons, largement " étrangers " aux problématiques européennes. Aux côtés des obstacles renvoyant à des enjeux de savoirs (langues, connaissances du droit étranger, etc.), des facteurs structurels apparaissent déterminants dans l'explication des résistances. Dans le cas des juges français, l'Europe apparaît souvent comme lointaine et complexe. Du fait de ces représentations, les juges hésitent à mobiliser les instruments de coopération comme Eurojust, car ceux-ci ne répondent pas, selon eux, à leurs objectifs d'efficacité imposés par un système national caractérisé de plus en plus par une productivité rationalisée ${ }^{77}$. Comme le souligne un 
juge : « Si l'on a cent dossiers dans son cabinet, il faut traiter les dossiers rapidement, ce que la prise en compte de la dimension européenne ne permet pas ${ }^{78}$ ". Pour de nombreux policiers, la résistance des magistrats nationaux s'explique également par la «culture » de travail de ces derniers :

« je crois que c'est dans leur culture de travail, parce que les policiers ont plus un travail de groupe que les magistrats, et donc on a plus cette culture de travailler ensemble, c'est ancestral, on n'a pas attendu l'Europe. Pour eux [les magistrats], au contraire la majorité du travail se fait de façon individuelle et indépendante ${ }^{79}$ ».

Face à ce constat, il semble que les relations personnelles et informelles demeurent des voies de recours privilégiées pour les juges qui, volontairement ou non, sont confrontés à une affaire transnationale : «il est évident que quand vous connaissez directement les personnes du niveau européen ou même de l'autre pays, parce que vous avez déjà collaboré par exemple, cela facilite les échanges. On n'hésite pas trop ${ }^{80}$ ". Dans ces conditions, le travail des membres d'Eurojust consiste " à sensibiliser les juges sur notre unité et surtout à leur montrer que nous sommes efficaces. Mais il faut du temps et des résultats ${ }^{81}$ ". L'exemple du MAE illustre également cette double dynamique d'apprentissage et de résistance. A la différence d'instruments comme Eurojust ou les magistrats de liaison, le MAE a été très rapidement utilisé par les juges. Le fait que ce dispositif ait remplacé l'ancienne procédure et qu'il représente ainsi le nouvel outil juridique d'extradition constitue la première explication de cette " réussite ». Cette utilisation quotidienne, à la vue des statistiques des différentes institutions nationales et européennes, ne signifie pas que ce dispositif a été totalement admis. Au-delà du poids des pratiques quotidiennes (rédaction, traduction, éléments justificatifs), dans le cas de cette nouvelle procédure d'extradition, les résistances portent essentiellement sur son contenu juridique et normatif ${ }^{82}$.

Enfin, si les acteurs précédemment évoqués occupent de façon plus ou moins constante des places centrales dans le champ de la coopération pénale européenne, d'autres, à l'inverse, se trouvent relégués à la périphérie. Les acteurs comme les ONG, les syndicats et les Parlements - européen et nationaux - restent largement à la marge du champ pénal européen. Cette mise à l'écart dans l'institutionnalisation de la coopération judiciaire s'explique par les logiques juridiques, politiques et sociales du champ de la sécurité européenne dans lequel s'inscrit celui de la coopération pénale ${ }^{83}$. Néanmoins, en matière de coopération judiciaire, le Parlement européen tente, malgré un rôle exclusivement consultatif en matière de justice, de s'investir par l'intermédiaire de certaines prises de position concernant la question du contrôle budgétaire et de la fraude aux intérêts communautaires ${ }^{84}$. La question des droits fondamentaux et de leur respect représente un second point d'entrée pour le Parlement européen qui coïncide avec un renforcement institutionnel dans le processus décisionnel européen ${ }^{85}$. Les ONG et les syndicats restent, eux aussi, encore largement à la périphérie du champ malgré des initiatives répétées pour s'y imposer à travers la prise en compte des enjeux qui structurent la coopération judiciaire européenne. A l'origine de différentes déclarations, l'Association européenne des magistrats pour la démocratie et la liberté (MEDEL) tente de s'investir dans le champ en s'inscrivant dans la filiation des premières initiatives de magistrats ${ }^{86}$. Concernant les $\mathrm{ONG}$, il semble qu'on assiste à une prise de position de plus en plus visible de certaines d'entre elles sur le terrain du Judiciaire. Des organisations comme JUSTICE, Statewatch et Amnesty Europe développent de plus en plus un travail relatif aux conséquences normatives de la coopération pénale européenne. Si cette évolution est encore difficilement mesurable dans le secteur 
pénal, les analyses produites par Adrian Favell dans son étude des ONG investies dans les politiques d'immigration européennes constituent des hypothèses pertinentes ${ }^{87}$. A côté des magistrats, les membres de ces organisations deviennent des collaborateurs de plus en plus sollicités par la Commission et le Parlement européen en tant que spécialistes des questions judiciaires.

Une meilleure connaissance de ces relations permet d'expliquer que, dans le cas de la coopération pénale, la logique d'harmonisation induisant une uniformisation des systèmes nationaux est généralement repoussée. Les projets d'harmonisation dans le domaine judiciaire (Corpus Juris, Procureur européen, harmonisation des procédures pénales) sont le plus souvent bloqués par l'utilisation de la méthode intergouvernementale et des règles d'unanimité qui, dans le cas de la création de l'unité Eurojust, ont prévalu. La logique de convergence est privilégiée au détriment de l'harmonisation, ce qui aboutit à des relations de concurrence entre les institutions selon qu'elles sont structurées dans une démarche de convergence ou d'harmonisation. Les conflits institutionnels entre l'OLAF, Eurojust, les magistrats de liaison et Europol prennent place dans une telle configuration. Le fait qu'il n'existe actuellement entre ces institutions que des accords ou mémorandums "vides de sens ${ }^{88}$ " illustre cette logique conflictuelle dominante. Ce type de relation engendre des conséquences au niveau de la normativité du régime pénal qui se met en place à l'échelle européenne. Si l'étude des relations de pouvoir qui traversent le champ de la coopération pénale européenne permet de comprendre les principales dynamiques sociales et politiques de transformation, la question du changement se pose également au niveau des représentations des acteurs de ce champ.

La légitimation de la coopération judiciaire européenne comme "solution politique "

41 L'intérêt d'articuler de façon équilibrée les idées, les intérêts et les institutions, en accordant une véritable place à la dimension cognitive, réside dans le fait de pouvoir interroger directement les processus de légitimation. La sécurité, en l'espèce la coopération judiciaire européenne, ne doit donc pas être considérée comme une donnée objective, mais comme le résultat de croyances définies par les jeux institutionnels, politiques et sociaux. Notre but n'est pas de juger de la réalité de la " menace criminelle ", mais de comprendre dans quelle mesure le cadre cognitif ${ }^{89}$ qui érige les phénomènes criminels en enjeux politiques de premier ordre pèse sur les stratégies et les décisions des acteurs, et donc sur les instruments mis en œuvre dans le champ pénal européen. Quels que soient les choix politiques, ces derniers se construisent autour d'un " récit 90 ", dont le but (dans une logique fonctionnaliste) est de rendre intelligibles des situations extrêmement complexes et de stabiliser les représentations à partir desquelles les orientations seront arrêtées. Nées des interactions de multiples acteurs, les dynamiques cognitives tendent peu à peu à s'autonomiser et à s'imposer comme représentation dominante. Il s'agit de retracer les processus de problématisation grâce à la compréhension des différentes étapes et niveaux où ces problèmes de lutte contre " les criminalités transnationales » se sont construits. Le danger réside ici dans une trop grande simplification du discours, lequel reste avant tout un objet complexe, avec des ruptures, des différences selon les conjonctures, les arènes et la position des acteurs.

Dans le domaine de la lutte contre les phénomènes criminels, on assiste à la constitution d'un « récit » introduisant une corrélation entre les représentations de la menace et une interprétation normative de type : " pour lutter contre l'ennemi 
transnational que sont les phénomènes criminels en tous genres, les Etats doivent coopérer ». Préconisée par un grand nombre d'observateurs opérationnels et institutionnels, cette recette de politique publique traduit, pour une part, une perte de confiance dans la puissance de l'Etat, jugé incapable de faire face individuellement à une menace criminelle ayant su, elle, tirer parti de la mondialisation. Un lien de causalité est établi entre l'ouverture des frontières et le développement de la criminalité transfrontalière. Devant la puissance présumée croissante des organisations criminelles, les pouvoirs publics se définissent comme déficients et se doivent de s'organiser pour être le plus efficaces possible. La rhétorique qui légitime la coopération est alors la suivante : l'ouverture des frontières a entrainé la multiplication des échanges et provoqué une augmentation de la criminalité transfrontalière dont l'action est facilitée par la disparition des contrôles. L'espace européen, découpé en autant de sous-espaces pénaux nationaux, est devenu de plus en plus désuet et faible, les textes relatifs à l'entraide judiciaire en matière pénale n'étant plus adaptés. Les délais d'extradition ou de renvoi des commissions rogatoires sont, par exemple, extrêmement longs. Les différences de réglementations entre pays de l'Union profitent aux organisations criminelles qui, elles, ne connaissent pas les frontières. Enfin, la mise en place d'une coopération judiciaire est également motivée par le souci d'accompagner et d'encadrer la coopération policière qui existe déjà, notamment avec Europol. La coopération étatique au niveau européen, et plus largement au niveau international, s'impose dès lors comme « la solution » aux yeux de la quasi-totalité des acteurs, qu'ils soient professionnels, politiques ou administratifs. Cette " solution » nécessite la mise en œuvre de politiques publiques particulières, avec notamment la construction d'organismes spécialisés. Comme le souligne un fonctionnaire européen, « on a utilisé la lutte contre le crime organisé qui est transnational par définition [...]. Et c'est une vérité absolue, contre le blanchiment d'argent qui est transnational, pour mettre en avant des progrès substantiels en matière de coopération nationale, c'est une évidence politique ${ }^{91} »$.

Si les premiers groupes de coopération policière comme le club de Berne, le groupe TREVI ou encore le club de Vienne se sont structurés pour l'essentiel à travers la lutte antiterroriste des années 1970 92, la coopération judiciaire s'est principalement organisée depuis le début des années 1990, en réponse au défi de la criminalité transfrontalière. Plutôt qu'une rupture cognitive, cette différence apparaît comme le résultat d'une labellisation toujours plus large de phénomènes criminels s'appuyant sur des représentations diverses et synthétiques « dans l'esprit d'une flexibilité maximale, qui conduit avant tout à rechercher l'efficacité, en matière de lutte contre des réseaux, des trafics ${ }^{93}$ ». Cette labellisation fait apparaître " la criminalité organisée » comme un phénomène global, avec de multiples vitrines qui, chacune à leur tour, peuvent devenir pour un certain temps dominantes, à l'image des réseaux illégaux d'immigration clandestine ou de prostitution pour lesquels la médiatisation joue un rôle central ${ }^{94}$. Comme le souligne Murray Edelman : " La structure cognitive ne peut demeurer crédible et opérante que si les ennemis du moment sont remplacés par d'autres; avec le temps et à mesure qu'évoluent les modes régissant la désignation des dangers, les ennemis se succèdent, bien que les nouveaux puissent aussi coexister avec les autres ${ }^{95}$. "Depuis les attentats du 11 septembre 2001, l'affirmation de la figure du terrorisme comme menace transnationale nécessitant le renforcement de la coopération judiciaire entre parfaitement dans cette logique. De façon générale, les perceptions du combat contre ce que l'on appelle « les organisations criminelles » se construisent dans le cadre 
de la globalisation économique, et notamment de l'intensification des flux financiers ${ }^{96}$. L'objectif premier de lutte contre l'argent sale de la drogue s'est progressivement étendu à la répression d'un ensemble plus vaste de délinquances économiques, communément désignées sous le terme de "White-Collar Criminality ${ }^{97}$ ", dans lequel l'UE s'engage officiellement le 8 novembre 1990, avec l'adoption par le Conseil de l'Europe de la «Convention relative au blanchiment, au dépistage, à la saisie et à la confiscation des produits du crime ».

S'intéresser aux logiques discursives permet, enfin, de démontrer que l'Union européenne ne constitue pas l'unique lieu du global, seul producteur de sens. Comme l'indique Yves Surel, les processus analysés à l'échelle européenne "ne relèvent pas spécifiquement de l'Union européenne mais cadrent plus généralement avec les dynamiques traditionnellement attachées à l'idée de globalisation ${ }^{98} »$. La question des représentations et de leurs circulations entre les différents niveaux de pouvoir matérialise cette dimension. A travers les interactions cognitives et donc sociales qui ont lieu au sein des organisations internationales (ONU, GAFI, G7/G8, Conseil européen) il est possible de retracer une partie de la circulation des représentations du monde à l'origine de la construction des enjeux et des recettes de l'action publique à l'échelle européenne. De la sorte, l'européanisation du Judiciaire est un élément du processus plus large d'une gouvernementalité internationale ${ }^{99}$.

Les effets normatifs de l'institutionnalisation du champ de la coopération pénale européenne

En guise de conclusion, l'étude de l'européanisation du domaine pénal à travers la compréhension de ses principales étapes et des logiques constitutives du champ de la coopération pénale européenne permet de poser, sous un angle critique, la question de la normativité. Le fait que le champ judiciaire soit traversé par des logiques de compétition/collaboration entre une multiplicité d'acteurs aux positionnements hétérogènes produit une accumulation de solutions aux orientations incertaines ${ }^{100}$. La superposition normative, résultat de jeux sociaux et politiques complexes au cœur du processus décisionnel européen, entraîne des répercussions d'ordre juridique. Face aux exigences de sécurité qui constituent le discours dominant des acteurs principaux du champ de la coopération pénale européenne, il semble que les questions relatives au respect des droits fondamentaux constitutionnels aient été reléguées à la marge ${ }^{101}$. Le témoignage d'un membre du SGCE illustre cette idée :

« Nous avons toujours pensé que l'un va avec l'autre et pas seulement pour le mandat d'arrêt européen. Notamment avec le principe de la reconnaissance mutuelle. Il faut un minimum standard et nous, on plaide pour cela depuis des années. Cela fait 4 ou 5 ans que l'on demande de développer ça, mais c'est extrêmement difficile. Pour faire une harmonisation du droit pénal, il suffit de décider ce qui est répréhensible ou non, tandis que pour le droit procédural c'est tout qui est mélangé et on a des systèmes extrêmement hétérogènes. Même quand on voit les petites propositions faites par la Commission ça cause de gros problèmes $102 »$.

L'exemple du MAE s'inscrit dans cette hypothèse, comme le montre le témoignage d'une représentante de la Direction générale JLS lors d'un séminaire relatif à cet instrument : « Il y avait une telle pression des Etats pour aller le plus vite possible lors des discussions, que nous n'avons pas pu défendre notre position et nos propositions sur la question des droits de la défense ${ }^{103}$ ». La mise en œuvre actuelle de ce dispositif pénal et la forte résistance des Cours constitutionnelles nationales sur la question du respect des libertés publiques attestent qu'il existe actuellement un déséquilibre 
structurel de l'espace pénal européen en défaveur des droits procéduraux et des libertés civiles ${ }^{104}$. Ce phénomène ne résulte pas d'une mauvaise volonté mais d'un effet de champ renvoyant à une multiplicité de jeux et d'arènes de décision. Largement dépendante des logiques dominantes du champ de la sécurité, la coopération pénale tend néanmoins à s'autonomiser comme le montrent les différentes contributions de ce numéro. Pourtant force est de constater que la dimension normative ne constitue pas encore la problématique dominante pour les acteurs décisionnels. Ainsi, contrairement à l'image d'une formalisation et d'une homogénéisation progressive des règles de l'UE, le champ de la coopération pénale européenne est largement marqué par des logiques informelles de coopération, des luttes institutionnelles et par l'omniprésence de l'agenda politique international et national.

\section{NOTES}

1.Delmas-Marty M., Les Forces imaginantes du droit : Le relatif et l'universel, Paris, Le Seuil, 2004, p 7.

2.Levasseur G., Chavanne A., Montreuil J., Droit pénal général et procédure pénale, 14e édition, Paris, Sirey, 2002, p 2.

3.Adopté le 22 avril 1996, par le Conseil « Justice et Affaires intérieures » de l'Union européenne, le dispositif des magistrats de liaison concerne, selon l'action commune, « un cadre d'échange de magistrats de liaison visant à l'amélioration de la coopération judiciaire entre les Etats membres de l'Union européenne ». Le 29 juin 1998, le Conseil européen adopte à Birmingham une autre action commune stipulant la création d'un Réseau judiciaire européen (RJE) dont l'objectif est également de faciliter l'échange d'informations entre les Etats membres sur le terrain judiciaire. Adopté politiquement lors du sommet de Tampere les 15 et 16 octobre 1999, l'unité de coopération Eurojust constitue la première unité judiciaire européenne multilatérale, marquant une véritable évolution institutionnelle par rapport aux systèmes des magistrats de liaison et du Réseau judiciaire européen.

4.Entretien réalisé avec un membre du Secrétariat général du Conseil européen en charge des questions de coopération judiciaire, mars 2004. Afin de respecter l'anonymat - souvent demandé - des personnes interviewées, nous ne donnerons, dans la majorité des cas, que la nationalité et/ou la fonction des personnes citées. 5.Entretien avec Adeline Hazan, députée européenne chargée des questions de justice, mars 2001. Sur ce point, voir également : Manifeste de Strasbourg, « Le parquet Européen, peut-on encore attendre? », Syndicat de la magistrature, octobre 2000. 6.Commissariat général du Plan, Quels avenirs pour l'Europe de la justice et de la police, rapport du groupe présidé par Patrick Hubert, Paris, La Documentation française, décembre 1999, p. 17.

7.En 1992, le traité de Maastricht crée le « 3e pilier » de l'Union, consacré exclusivement aux questions de «Justice et Affaires intérieures » et met en place les contours d'un système intergouvernemental, fondé sur le vote à l'unanimité et sur un pouvoir décisionnel aux mains des Etats. Depuis le programme de La Haye de 2004, la 
Direction générale «Justice et Affaires intérieures » (JAI) a été rebaptisée « Justice, Liberté et Sécurité » (JLS).

8.Dans l'article 30 du traité d'Amsterdam, afin « d'encourager la coopération [policière] par l'intermédiaire d'Europol et, en particulier, dans les cinq ans qui suivent la date d'entrée en vigueur du traité d'Amsterdam », le Conseil européen : «a) permet à Europol de faciliter et d'appuyer la préparation, et d'encourager la coordination et la mise en œuvre d'actions spécifiques d'enquêtes menées par les autorités compétentes des Etats membres, y compris des actions opérationnelles d'équipes conjointes, comprenant des représentants d'Europol à titre d'appui ; b) arrête des mesures destinées à permettre à Europol de demander aux autorités compétentes des Etats membres de mener et de coordonner leurs enquêtes dans les affaires précises, et de développer des compétences spécialisées pouvant être mises à la disposition des Etats membres pour les aider dans des enquêtes sur la criminalité organisée [...] ». A travers ces deux orientations, se dessine la dimension opérationnelle qui constitue, selon certains policiers investis dans la coopération européenne, l'étape naturelle dans le développement d'Europol. Même si aujourd'hui cette dimension opérationnelle n'a toujours pas abouti, pour certains fonctionnaires européens ou représentants nationaux ayant participé aux discussion de l'époque, l'idée de donner un pouvoir opérationnel à Europol a servi indéniablement de justification pour ceux qui désiraient mettre l'entraide en matière de justice au cœur des discussions.

9.Voir entre autres : Fontanaux D., « La coopération judiciaire en Europe », Problèmes politiques et sociaux, Paris, La Documentation française, $\mathrm{n}^{\circ} 786,26$ juillet 1997 ; Gautron J.-C., Droit européen, coll. « Mementos » Dalloz, 9e éd., 1999 ; Labayle H., « La coopération européenne en matière de justice et affaires intérieures et la CIG », Revue trimestrielle de droit européen, Paris, janvier-mars 1997, pp 1-35 ; Barbe E., Justice et Affaires intérieures dans l'Union européenne : un espace de liberté, de sécurité et de justice, Paris, La Documentation française, 2002 ; De Kerchove G., Weyembergh A., L'Espace pénal européen : enjeux et perspectives, Bruxelles, Editions de l'université de Bruxelles, 2002.

10.Nous utiliserons dorénavant dans l'article le signe MAE pour désigner le Mandat d'arrêt européen.

11.Veyne P., Comment on écrit l'histoire. Essai d'épistémologie, Paris, Le Seuil, 1971. 12.Pour une analyse du projet Corpus Juris, voir l'article de Natacha Paris dans ce numéro. Voir également, Manacorda S., « Pour un Corpus Juris, perspectives d'unification du droit pénal des affaires en Europe », Les Cahiers de la sécurité intérieure, 36, 2nd trimestre 1999, pp. 15-23.

13.« Corpus Juris portant dispositions pénales pour la protection des intérêts financiers de l’Union européenne », Paris, Recueil Dalloz, 1re éd., 1997.

14.Ibid, article 18, al.1, 1997.

15.Ibid, article 19. § 2.

16.Entre autres : Delmas-Marty M., Giudicelli-Delage G., Lambert-Abdelgawad E. (dir.), L'Harmonisation des sanctions pénales en Europe, Paris, Société de législation comparée, 2003 ; Delmas-Marty M, « Union européenne et droit pénal », Cahiers de droit européen, Bruxelles, 33e année, $\mathrm{n}^{\circ} 5$ et 6, pp. 607-653, 1997 ; Delmas-Marty M., Procédures pénales d'Europe : Allemagne, Angleterre et Pays de Galles, Belgique, France, Italie, Paris, Presses universitaires de France, 1995.

17.Voir notamment les travaux menés à l'université de Nancy-II et à la Faculté de droit et des sciences sociales de Poitiers. 
18.La création du MAE a engendré, dans le champ scientifique, un grand nombre d'études essentielles concernant les conséquences juridiques et normatives d'un tel dispositif, en particulier sur la question du respect des libertés publiques. Voir notamment les travaux menés par le CEPS (Center for European Policy Studies), dans le cadre du programme européen Challenge, sur la transposition du MAE dans les ordres juridiques des Etats membres, Constitutional Challenges to the European Arrest Warrant, ed. Wolf Legal Publishers, Nijmegen, juin 2006. Voir également Blextoon R. (dir.), Handbook on the European Arrest Warrant, Cambridge, TMC Asser Press, 2005 ; ERA-Forum scripta iuris europaei, Académie de Droit européen de Trèves, $n^{\circ}$ 4, 2003. 19.Partant du postulat qu'il est nécessaire d'analyser les logiques sociales structurant la mise en place de la jurisprudence communautaire, certains auteurs évoquent les dynamiques transnationales ou inter-court competition, afin d'expliquer la participation active des juges nationaux aux cotés de la CJCE dans la construction d'un droit intégré. Voir notamment : Dehousse R., « L'Europe par le droit : plaidoyer pour une approche contextuelle », Politique européenne, 2000-04, n 1, pp. 63-83 ; Alter K., « The European Court's Political Power », European Yearbook of Comparative Government and Public Administration, 1996, vol.3, pp. 241-246 ; Stone Sweet A., Caporaso J., « La Cour de justice et l'intégration européenne », Revue française de science politique, vol.48, 1998, pp. 194-244 ; Burley A.-M., Mattli W., « Europe before the Court : a political theory of legal integration », International Organization, 47 (1), hiver 1993, pp. 41-76.

20.Commaille J., Réseau Europe Droit et Société, Barcelone, Institut de sciences sociales et politiques, 2000, p. 59.

21.Lascoumes P., Le Galès P. (dir.), Gouverner par les instruments, Paris, Presses de Sciences Po, 2004, p. 12.

22.Ibid, p. 12

23.Stone Sweet A., Sandholtz W., «European Integration and Supranational Governance», Journal of European Public Policy, septembre 1997, vol.4, n³ 3, 1997, pp. 297-317.

24.Voir l'article de Maik Martin dans ce numéro.

25.Radaelli C., « The Domestic Impact of European Union Public Policy : Notes on Concepts, Methods, and the Challenge of Empirical Research ", Politique européenne, $n^{\circ} 5,2001$, p. 110. « Europeanization refers to : Processes of (a) construction, (b) diffusion and (c) institutionalization of formal and informal rules, procedures, policy paradigms, styles, 'ways of doing things' and shared beliefs and norms which are first defined and consolidated in the making of EU policy and politics and then incorporated in the logic of domestic discourse, identities, political structures and public policies ».

26.Entre autres : Caporaso J., Cowles M., Risse T. (dir.), Transforming Europe, Europeanization and Domestic Change, Ithaca, Cornell University Press, 2001 ; Börzel T., Risse T., « When Europe Hits Home : Europeanization and Domestic Change », European Integration online Papers (EIoP), 2000 : http://eiop.or.at/eiop/texte/ 2000015a.htm>http://eiop.or.at/eiop/texte/2000-015a.htm.

27.Pour une définition de la notion d'européanisation : Radaelli C., op. cit, pp. 107-142 ; Palier B., Surel Y. et al, L'Europe en action : analyses d'européanisation, Paris, L'Harmattan, 2006 (à paraître). Pour une étude théorique et empirique des politiques intergouvernementales à partir de la notion d'européisation, Mégie A., Ravinet P., « Processus intergouvernementaux et européanisation : La construction des espaces 
européens de l'enseignement supérieur et de la justice », in Palier B., Surel Y. et al, op. cit.

28.Créé en 1999 à la suite du scandale de la commission Santer, en remplacement de l'Unité de coordination de la lutte anti-fraude (UCLAF), l'OLAF constitue une institution communautaire chargée de lutter contre les fraudes aux intérêts financiers communautaires. En 2000, dans une logique de judiciarisation amorcée depuis quelques années, une unité de magistrats est mise en place en son sein. Par leur nature protéiforme, certains délits ou détournements peuvent relever à la fois du 1er pilier (domaine de compétence de l'OLAF) et du 3e pilier (domaine de compétence des services nationaux et, depuis fin 2001, de l'unité judiciaire Eurojust). Voir l'article de Véronique Pujas dans ce numéro.

29.Si l'approche néo-fonctionnaliste met l'accent sur l'émergence d'un espace d'action publique au niveau européen, l'approche intergouvernementaliste, dont l'un des auteurs principaux est Andrew Moravchick, définit le processus européen comme le résultat des jeux de négociations, «bargains », entre les Etats membres. La construction de l'Union européenne serait ainsi totalement dépendante des stratégies et ressources des Etats engagés dans les négociations. La plupart des travaux récents consacrés aux politiques européennes débutent généralement par une remise en cause de ces deux approches classiques du processus européen, leur reprochant leurs visions théoriques et normatives de la construction européenne, qui les amènent à ne pas prendre en compte la complexité des processus à l'œuvre. Voir entre autres : Featherstone K., Radaelli C., The Politics of Europeanization, Oxford, Oxford University Press, 2003; Lequesne C., Smith A. (dirs.) Cultures \& Conflits, « Interpréter l'Europe : éléments pour une relance théorique ", Paris, l'Harmattan, n² 28, hiver 1997.

30.Palier B., Surel Y., et al, op. cit.

31.Protocoles additionnels à la Convention européenne d'entraide judiciaire en matière pénale, Strasbourg, 17. III, 1978.

32.La France ne ratifiera cette Convention qu'en 1987.

33.Fontanaud D., « Les accords de Schengen », Problèmes politiques et sociaux, Paris, La Documentation française, $n^{\circ}$ 763-764, 15 mars 1996 ; Domenach J., « La sécurité intérieure en Europe », Clefs, Montchrestien, 1998.

34.Les premiers échanges de magistrats eurent lieu entre les Pays-Bas et la France, ainsi qu'entre la France et l'Italie. Si entre les Pays Bas et la France, un magistrat de liaison fut nommé par chaque pays, en revanche entre l'Italie et la France, seul un magistrat français fut envoyé à cette époque.

35.Le Conseil « Justice et Affaires intérieures » de l'Union européenne adopte, le 22 avril 1996, une action commune sur la base de l'article K.3 du traité de Maastricht " concernant un cadre d'échange de magistrats de liaison visant à l'amélioration de la coopération judiciaire entre les Etats membres de l'Union européenne ».

36.La communautarisation des domaines asile-immigration et coopération judiciaire civile s'est accompagnée d'un renforcement de la place de la Commission et du Parlement européen (Titre I du traité d'Amsterdam). Parallèlement, de nouveaux instruments et principes juridiques sont introduits comme la décision, la décisioncadre, et le concept « d'espace européen judiciaire » (Titre IV du traité d'Amsterdam). Sur le domaine " asile et immigration », voir notamment : Cultures \& conflits, « La mise à l'écart des étrangers : la logique du visa Schengen », $n^{\circ} 49$ et 50, Paris, l'Harmattan, Paris, 2003 ; voir également : Piazza P., « Politique d'immigration et d'asile, compte rendu du troisième Congrès européen des juristes spécialistes des questions 
d'immigration et d'asile en Europe », Les Cahiers de la sécurité intérieure, n 55 , 1er trimestre 2004, pp. 259-273.

37.Traité d'Amsterdam, Titre IV, article 31 (ex-article K. 3).

38.Voir l'article de Michel Mangenot dans ce numéro.

39.Les magistrats de liaison constituent d'ailleurs la grande majorité des points de contacts du Réseau judiciaire européen.

40.Conclusions de la présidence, Conseil européen de Tampere, 15 et 16 octobre 1999.

41.Extrait du traité sur l'Union européenne (1992) modifié par le traité d'Amsterdam (1997), Titre VI, article 29 (ex-article K.1).

42.Conclusions de la présidence, Conseil européen de Tampere, 15 et 16 octobre 1999, article 46.

43.Entretien réalisé avec un membre du Secrétariat général du Conseil européen, avril 2004.

44.Ibid.

45.Conclusions de la présidence, Conseil de Tampere, article 35. Voir sur cette problématique : Cultures \& conflits, « De Tampere à Séville : bilan de la sécurité européenne », Bigo D., Guild E. (dir.) n 45-46, Paris, l'Harmattan, printemps 2002. 46. Mégie A., « Le 11 septembre : élément accélérateur de la coopération judiciaire européenne ? », Les Cahiers de la Sécurité Intérieure, n 55, premier trimestre 2004, pp. 91-120.

47.Entretien avec un fonctionnaire européen en charge de la rédaction de la proposition de MAE, DG JAI, mai 2004.

48.Ibid.

49.Ibid.

50.Sur la mise en œuvre juridique du MAE, voir la contribution de Maik Martin dans ce numéro.

51.Pour une analyse du Programme de La Haye : Bigo (D.), « Liberty, whose liberty? The Hague Programme and the conception of freedom », Challenge, http:// www.libertysecurity.org/article339.html

52.Cultures \& Conflits, « Sociologie de l'Europe », Guiraudon V. (dir.), Paris, l'Harmattan, n 38-39, été/automne 2000. Voir également: Georgakakis D. (dir), Les Métiers de l'Europe politique : acteurs et professionnalisations de l'Union européenne, Strasbourg, Presses universitaires de Strasbourg, 2002.

53.Lequesne C., Smith A., op. cit., p. 86.

54.Palier B., Surel Y., "L'analyse politique de l'action publique : confrontation des approches, des concepts et des méthodes», Revue française de science politique, vol.55, $\mathrm{n}^{\circ} 1,2005$.

55.Dehousse R., Majone G., «The Dynamic of Integration : from the Single European Act to the Maastricht Treaty», in Martin S. (dir), The Construction of Europe - Essays in Honor of Emile Noël, Dordrecht, Kluwer, pp. 91-112, 1994.

56.Nous reprenons ici le concept de « champ » défini par Pierre Bourdieu comme une configuration, un espace de luttes, de relations objectives entre des positions qui s'imposent objectivement à leurs occupants, agents ou institutions. Voir : Bourdieu P., Réponses, Paris, Le Seuil 1992, également Bourdieu P., Choses dites, Paris, Editions de minuit, 1987.

57.Bigo D., «La mondialisation de l'(in)sécurité ? Réflexions sur le champ des professionnels de la gestion des inquiétudes et analytique de la transnationalisation des processus d'(in)sécurisation », Cultures \& Conflits, n 58, 2005, p. 74. 
58.Ibid, p. 73.

59.Dans son article concernant la constitution de l'unité Eurojust, Michel Mangenot montre la manière dont le leadership institutionnel du Secrétariat général du Conseil varie dans le temps et n'engendre pas systématiquement une concrétisation des projets. Alors que le projet Eurojust soutenu par cette institution se concrétise, une autre proposition relative à la création d'une grande école de la magistrature européenne est quant à elle refusée par les Etats membres.

60.Putnam R., « Diplomacy and Domestic Politic s: The Logic of Two-Level Games », International Organisation, 42, 1988, pp. 427-460.

61.Au sens développé par Howard Becker concernant « les entrepreneurs moraux », in Becker H., Outsiders, The Free Press of Glencoe, 1963, Trad. Paris, Editions Métailié, Paris, 1985.

62.Entretien réalisé avec un membre de l'unité de magistrats de l'OLAF, février 2001. 63. Comme le souligne un fonctionnaire européen ayant participé aux étapes d'institutionnalisation de l'espace pénal européen : « ces différentes décisions sont le résultat de jeux d'influence politique au sein même des Etats entre les différents ministères ». Entretien avec un ancien membre français du Secrétariat général du Conseil européen, février 2001.

64." Chaque Etat veut donner une inflexion parce qu'il ne veut pas que le projet s'oriente dans un certains sens. C'est souvent le premier qui sort qui gagne, ou du moins, qui imprime une inflexion, une orientation générale lors du Conseil européen. C'est donc pour ça que l'on assiste à des coalitions [...] on est effectivement dans des jeux de rapport de force. Lorsque la discussion achoppe sur une question, c'est donnant-donnant, un bras de fer, si tu acceptes ça, j'accepte ça ». Entretien avec un ancien membre français au Secrétariat général du Conseil européen, février 2001. 65.De Maillard J., Fouilleux E., Smith A., « Technical or political ? : the working groups of the EU Council of Ministers ", Journal of European Public Policy, vol.12, $\mathrm{n}^{\circ} 4,2005$, pp. 609-623.

66.Voir l'article de Michel Mangenot dans ce numéro.

67.Mégie A., op. cit.

68.Livre vert de la Commission européenne sur le projet de Procureur européen. 69. Conclusions du Conseil européen de Tampere, op. cit.

70.Voir les articles de Véronique Pujas et de Natacha Paris dans ce numéro

71.Des programmes cadres tels que Falcone, Oisin II ou AGIS constituent des dispositif dont l'objectif est de financer le développement et l'évaluation des politiques publiques européennes, ainsi que d'encourager le renforcement de la coopération européenne. Le programme le plus récent AGIS couvre la période 2003-2004. Adopté le 22 juillet 2002, il a ainsi pour objectif « de favoriser la coopération policière et judiciaire en matière pénale et de soutenir l'apport des praticiens au développement de la politique européenne dans ce domaine ».

72.Georgakakis D., op. cit, p. 19.

73.Ibid, p. 30.

74.Sur ce type de mobilisations voir l'article de Natacha Paris dans ce numéro.

75. Becker H., op. cit.

76.Voir la contribution d'Amandine Scherrer dans ce numéro, sur les groupes d'experts dans le domaine de la sécurité et plus spécifiquement dans celui de la lutte antiblanchiment. Voir également sur la construction d'une expertise judiciaire, l'étude d'Antoine Vauchez sur l'institutionnalisation d'une « nouvelle justice » en Italie : 
Vauchez A., L'institution judiciaire remotivée : le processus d'institutionnalisation d'une "nouvelle justice" en Italie : 1960-2000, Paris, LGDJ, 2004.

77.Sur cette question voir notamment : Bastard B., Mouhanna C., Ackermann W., Une justice dans l'urgence. Le traitement en temps réel des affaires pénales, Mission de recherche Droit et Justice, juillet 2005.

78.Entretien avec un magistrat français, Paris, juin 2005.

79.Entretien avec le directeur adjoint d'Europol, mai 2001.

80.Entretien avec un magistrat en poste au ministère français de la Justice, mai 2004.

81.Entretien avec un membre d'Eurojust, avril 2001.

82.Voir l'article de Maik Martin dans ce numéro relatif aux arrêts des Cours anglaises, irlandaises et allemandes concernant la mise en œuvre du MAE dans les systèmes judiciaires nationaux.

83.Cultures \& Conflits, « De Tampere à Séville : bilan de la sécurité européenne », op. cit.

84.Voir les articles de Véronique Pujas et de Natacha Paris dans ce numéro

85.Rapport du Parlement Européen, « Recommandations au Conseil pour l'évaluation de la mise en œuvre du mandat d'arrêt européen », 2005.

86.Pour une analyse détaillée de ce groupe : Paris N., « Corpus Juris, une tentative controversée de lutte contre les fraudes communautaires », in Michel H., Paris N., Mangenot M., Mobilisation contre la « criminalité organisée » et institutionnalisation d'un espace judiciaire pénal européen (1996-2001), collection « Arrêt sur recherche » de la mission Droit et justice, 2005.

87.Favell A., «L'européanisation ou l'émergence d'un nouveau “champ politique" : le cas de la politique d'immigration ", Cultures \& Conflits, n 38-39, 2000, pp. 153-185. 88.Entretien avec un fonctionnaire européen, membre de l'unité des magistrats de l'OLAF, mars 2004.

89.Au sens de cadres d'interprétation du monde. Voir sur ce point : Muller P., « Les politiques publiques comme vision du monde», Faure A., Pollet G., Warin P. (dir.), La Construction du sens dans les politiques publiques, débats autour de la notion de référentiel, Paris, L'Harmattan, 1995 ; également « Les approches cognitives des politiques publiques ", Revue française de science politique, vol.50, n² 2, avril 2000. 90.Radaelli C., « Logique de pouvoirs et récits dans les politiques publiques de l'Union Européenne », Revue française de science politique, vol.50, n² 2, avril 2000, pp. 255-275. 91.Entretien avec un fonctionnaire européen, membre de l'unité de magistrat de l'OLAF, février 2001.

92.Pour une présentation complète de la constitution des premiers groupes européens de coopération policière : Bigo D., Police en réseaux, Paris, Presses de Sciences Po, 1996. 93.Entretien avec un magistrat français membre d'Eurojust, mai 2001.

94.Si un cargo d'immigrés clandestins échoue sur les côtes européennes, l'événement ravivera immédiatement à la une des médias le débat sur les réseaux criminels à l'origine de ces trafics.

95.Edelman M., Pièces et règles du jeu politique, The University of Chicago, Chicago, 1988, Trad. Paris, Seuil, 1991, p. 156.

96. Chesnais F., « Blanchiment de l'argent sale et mondialisation financière », Relations internationales et stratégiques, n²0, hiver 1995, pp. 145-153.

97. Criminalité en col blanc ».

98.Surel Y., «L'approche cognitive de l'intégration européenne », Revue française de science politique, vol.50, $\mathrm{n}^{\circ} 2$, avril 2000, p. 251. 
99.Voir la contribution d'Amandine Scherrer sur le rôle du G7/G8 dans la création d'un « régime global » de lutte anti-blanchiment. Voir également les articles de Natacha Paris et de Véronique Pujas qui interrogent les effets discursifs et institutionnels que la lutte contre la criminalité financière a produit sur le champ pénal européen.

100.Voir l'article dans ce numéro de Jacqueline Domenach relatif aux conséquences du processus d'européanisation judiciaire sur la réalité normative du régime pénal européen.

101.Voir notamment, Guild E., «L'état d'exception, le juge, l'étranger et les droits de l'Homme : trois défis des Cours britanniques », Cultures \& Conflits, $n^{\circ} 58,2005, \mathrm{pp}$.

183-203.

102.Entretien avec un membre du secrétariat général du Conseil Européen, mai 2004. 103.Déclaration d'une représentante de la Direction Générale (JLS) lors d'un séminaire relatif au Mandat d'arrêt européen, séminaire du CEPS (Center for European Policy Studies), Bruxelles, 13 février 2006.

104.Justice, Preliminary Response to the Commission Green Paper on Procedural Safeguards for Suspects and Defendants in Crimial Proceedings throughout the European Union, Avril 2003 ; Actes du séminaire "Justice and Home Affairs - Towards the Full Implemetation of the Amsterdam Treaty", organisé par la Fondation Cicero, Paris, 14 et 15 Février 2002.

\section{RÉSUMÉS}

L'objet de l'article est de tracer les pistes d'étude qui permettent de mieux comprendre les caractéristiques et les conséquences de la mise en place d'une coopération pénale à l'échelle européenne. Prenant en considération la littérature juridique et institutionnelle déjà importante sur ce sujet, il s'agit d'expliquer en quoi une approche historique en termes de processus constitue une première piste essentielle pour appréhender les différentes étapes de la construction d'un pouvoir pénal européen qui, loin d'être linéaire, apparaît plutôt comme intermittente et chaotique. Saisir les différentes phases de cette européanisation implique de travailler sur les moments de ruptures et de changements. La mise en évidence de ces instants critiques conduit, dans un second temps, à placer au cœur de l'analyse les interactions entre les acteurs via l'étude de leurs ressources sociales et de leurs représentations. La compréhension des logiques du champ de la coopération pénale permet, enfin, de donner du sens à la forme normative que revêt aujourd'hui le régime judiciaire européen notamment concernant le déséquilibre structurel qui existe en défaveur des droits procéduraux et des libertés civiles.

This article aims to trace new avenues of research that would allow a better understanding of the characteristics and consequences of the establishment of a judicial cooperation at the European level. We will show why the historical approach in terms of process may constitute the first essential avenue of research in order to seize the various stages of the construction of a European judicial power that appears more sporadic and chaotic than linear. The observation of the different stages implies working on the moments of ruptures and changes. Emphasizing critical moments leads to placing the interactions between actors at the heart of our analysis via the study of their social resources and representations. Lastly, the understanding of the logics of this 
field enables to give a meaning to the normative form of the contemporary European regime particularly with regards to the structural unbalance existing in disfavor of procedural rights and civil liberties.

INDEX

Index géographique : Union européenne

Mots-clés : coopération internationale, généalogie/ socio-génèse, juge, politiques publiques

\section{AUTEUR}

ANTOINE MÉGIE

Antoine Mégie est doctorant en science politique à l'IEP de Paris. Achève actuellement une thèse, sous la direction de Renaud Dehousse, portant sur la construction de la coopération pénale européenne à travers l'étude des principaux dispositifs d'entraide que sont l'unité Eurojust et le MAE. 